\title{
ENSAYO
}

\section{LOS PAÍSES EN DESARROLLO EN EL RÉGIMEN MULTILATERAL DE COMERCIO}

\author{
Magdalena María Núñez Jaramillo \\ Magíster en Derecho Internacional Económico \\ y Magíster en Relaciones Internacionales y Diplomacia \\ Segundo Secretario del Servicio Exterior Ecuatoriano \\ wmaggie_nj_80@yahoo.com
}

Recibido 10/03/17

Aceptado 22/10/17

\section{RESUMEN}

La Organización Mundial de Comercio (OMC) es la institución que ejecuta el régimen internacional del comercio que se configuró luego de finalizada la Segunda Guerra Mundial como uno de los mecanismos para crear las condiciones económicas y políticas que permitan evitar futuros enfrentamientos. Los países en desarrollo enfrentaron varias limitaciones al inicio del GATT y paulatinamente fueron consiguiendo importantes conquistas que les permitieron participar en el sistema multilateral de comercio de manera más justa y equitativa. El Trato Especial y Diferenciado (TED) y el Sistema Generalizado de Preferencias (SGP), amparados por la Cláusula de Habilitación, fueron los avances más importantes conseguidos por los países en desarrollo en el marco del GATT. Con la creación de la OMC, dichas conquistas se fueron consolidando e institucionalizando. La denominada Ronda de Doha para el Desarrollo, que inició en 2001, incorporó varias iniciativas positivas para los países en desarrollo y fortaleció el TED. Desafortunadamente, dicha ronda de negociaciones presenta un estancamiento desde hace varios años, lo que ha incidido en la proliferación de acuerdos comerciales bilaterales, regionales e interregionales que bien podrían mermar al sistema multilateral de comercio. Los países desarrollados han expresado su interés para que las negociaciones en la Ronda de Doha terminen por la falta de consenso y, en su lugar, se aborden nuevos temas en la agenda multilateral que respondan a las necesidades del mundo actual. Esto podría significar un importante retroceso en la participación de los países en desarrollo en el régimen multilateral de comercio.

Palabras clave: Países en desarrollo; trato especial y diferenciado, Ecuador. 


\title{
DEVELOPING COUNTRIES IN THE MULTILATERAL, TRADE REGIME
}

\begin{abstract}
The World Trade Organization (WTO) is the institution that implements the international trade regime that was set up after the end of World War II as one of the mechanisms to create the economic and political conditions to avoid future wars. Developing countries faced a number of constraints under the GATT and gradually achieved important gains that allowed them to participate in the multilateral trading system more fairly and equitably. Special and Differential Treatment (SDT) and the Generalized System of Preferences (GSP), covered by the Enabling Clause, were the most important achievements of developing countries under the GATT. With the creation of the WTO such achievements were consolidated and institutionalized. The so-called Doha Development Round, which began in 2001, incorporated a number of positive initiatives for developing countries and strengthened the SDT. Unfortunately, this round of negotiations has deadlocked for several years, which has contributed to the proliferation of bilateral, regional and interregional trade agreements that could undermine the multilateral trade system. The developed countries have expressed their interest in concluding the negotiations in the Doha Round due to the lack of consensus and, instead, addressing new issues on the multilateral trade agenda that respond to the needs of today's world. This could mean a significant decline in the participation of developing countries in the multilateral trading system.
\end{abstract}

Keywords: Developing countries; Special and Differential Treatment, Equator

\section{INTRODUCCIÓN}

El presente ensayo tiene como propósito hacer una revisión de la participación de los países en desarrollo en el sistema multilateral de comercio que se configuró luego de terminada la Segunda Guerra Mundial, así como los principales beneficios y limitaciones que dicho sistema les habría proporcionado con miras a la potenciación de su desarrollo económico y social.

Se iniciará el análisis con un repaso sucinto de los principales postulados de la teoría de los regímenes internacionales, los cuales servirán de base para explicar el surgimiento y funcionamiento del sistema multilateral de comercio y la institución en torno a la cual se organiza dicho sistema. Posteriormente, se expondrán los principales logros conseguidos por los países en desarrollo desde el surgimiento del régimen internacional de comercio en el marco del Acuerdo General sobre Aranceles Aduaneros y Comercio (GATT) hasta la configuración de la Organización Mundial de Comercio (OMC), así como algunas limitaciones y desafíos que, asimismo, han debido enfrentar. Finalmente, se plantearán algunas conclusiones sobre las perspectivas para los países en desarrollo en el sistema multilateral de comercio en los años venideros.

\section{El régimen internacional de comercio - La Organización Mundial de Comercio (OMC)}

\section{Configuración de los regímenes internacio- nales.}

Según Hasenclever, Mayer y Rittberger (1999), un régimen internacional es un conjunto de reglas, principios, procedimientos y normas uniformes para regular determinada actividad, 
en los cuales han coincidido los gobiernos con el fin de tornar más predecible el comportamiento de los Estados y evitar la adopción de decisiones unilaterales; este comportamiento basado en reglas generaría expectativas mutuas entre los países.

Para los referidos autores los regímenes internacionales pueden ser interpretados a la luz de premisas realistas, liberales o constructivistas, principales corrientes teóricas de las relaciones internacionales. Desde el realismo (específicamente desde la Teoría de la Estabilidad Hegemónica) es necesario el "liderazgo" de una potencia para que un régimen funcione; el líder o hegemón - mediante el uso de sus recursos e influencia - facilita la cooperación internacional y previene el incumplimiento de las normas. Desde la visión liberal, la "cooperación” sería la razón fundamental que permite la formación y el mantenimiento de los regímenes. Finalmente, desde el constructivismo, el "consenso ideológico" (conjunto de creencias, ideas y percepciones) es lo que haría posible la creación y el mantenimiento de los regímenes, los cuales serían una "construcción social".

La economía internacional liberal de postguerra fue basada en el liderazgo político y económico de los Estados Unidos (interpretación realista); sin embargo, el contexto mundial luego de la Segunda Guerra Mundial hacía latente la necesidad de fomentar la cooperación creciente (visión liberal), lo que no habría sido posible sin la configuración de nuevas ideas, creencias y percepciones sobre la necesidad de un nuevo orden mundial (aporte constructivista).

Según el realismo, los regímenes no están por encima ni fuera de la búsqueda del poder. Para Oatley (2004) los sistemas políticos tienden a reflejar los intereses de quienes los crearon, que son por lo general los Estados poderosos; estos construyen las instituciones políticas de acuerdo a sus intereses y éstas persisten mientras dichos países estén dispuestos a sostenerlas.
Para los liberales, los regímenes son neutrales, alteran el comportamiento de los Estados, producen efectos políticos y pueden tener funciones redistributivas; desde esta perspectiva para que un régimen funcione, los Estados deben tener intereses comunes que se beneficien con la cooperación (Keohane, 1993).

Por su parte, el constructivismo tiene una visión más positiva de los regímenes y considera que es posible cambiar elementos del sistema (Hasenclever, Mayer y Rittberger, 1999). La desastrosa experiencia de las guerras mundiales habría cambiado las percepciones o normas sociales de los países y los habría instado a crear una nueva "construcción social". Así, liderazgo, cooperación y consenso ideológico serían necesarios para la creación y el mantenimiento de un régimen internacional.

Según autores como Keohane y Nye (1989), las organizaciones internacionales son un instrumento de vinculación y convenientes para los Estados débiles. Por medio de las organizaciones, los Estados pequeños pueden aliarse y vencer la fuerza de los grandes; así, los países grandes deben buscar nuevas formas para ejercer su poder (surgimiento de un poder blando o Soft Law, según el cual la fuerza ya no es suficiente para conservar el poder, sino que intervienen otros factores como la dominación ideológica, cultural o económica) (Keohane y Nye, 1989). Con los regímenes, los países pequeños pueden coordinar sus acciones para alcanzar ganancias comunes (Attiná, 2001).

\section{La Organización Mundial de Comercio como régimen internacional}

Los postulados de la teoría de los regímenes internacionales permiten entender y analizar la Organización Mundial de Comercio (OMC) como la institución que ejecuta el régimen internacional del comercio. Puede considerarse que la OMC es el producto, en el ámbito comercial, de la creciente institucionalización del mundo experimentada luego de la Segunda Guerra Mundial. En ese contexto, los países se vieron en la necesidad de definir reglas, nor- 
mas e instituciones que regulen las relaciones internacionales en diferentes áreas, dando como resultado la creación de instituciones que garanticen la paz e incentiven las relaciones económicas internacionales.

Las consecuencias de la Guerra Mundial incentivaron la búsqueda de mecanismos para lograr una cooperación política, económica y social. Así, se crearon instituciones internacionales con el objeto de garantizar la paz y armonizar las relaciones económicas internacionales. La más importante de estas instituciones fue la Organización de las Naciones Unidas (ONU). Si bien el rol esencial de la ONU consistía en garantizar la paz, no obstante, en el marco de esta organización también se impulsaron objetivos de carácter económico tendientes a mejorar el bienestar y calidad de vida de todos los habitantes del mundo.

El fin de la guerra también coadyuvó para que se aunaran esfuerzos con miras a crear un nuevo orden económico internacional. Con esta finalidad se alcanzaron los denominados "Acuerdos de Bretton Woods", con los cuales fueron creados el Fondo Monetario Internacional (FMI), el Banco Mundial (ex BIRF) y se buscó también la creación de una Organización Internacional de Comercio (OIC), la cual no llegó a concretarse. Ante el fracaso de la OIC, se estableció el Acuerdo General sobre Aranceles Aduaneros y Comercio (GATT), acuerdo intergubernamental que servirá posteriormente de base para la creación de la OMC.

Estas instituciones buscaban propiciar un ambiente de cooperación en los ámbitos económico y político a fin de evitar futuros enfrentamientos. Como mecanismo para lograr este objetivo, en el marco de las referidas instituciones, se impulsó el compromiso para reducir la protección de los mercados y liberalizar el comercio.

La OMC, creada en 1995, está encargada de producir y regular las normas del comercio sobre la base de negociaciones, con miras a la liberalización del comercio y la reducción de los obstáculos que lo restringen. Sirve como foro para negociar acuerdos de comercio, así como para solucionar disputas comerciales entre los países miembros. En este sentido, según la Organización Mundial de Comercio (OMC, 2005), el principal propósito de dicha organización es contribuir a que las corrientes comerciales circulen con fluidez, libertad, equidad y previsibilidad.

Las reglas del régimen multilateral de comercio proveen el marco legal para las relaciones internacionales de comercio, especifican cómo los gobiernos se tratarán mutuamente y qué tipos de políticas comerciales se pueden o no adoptar. Las normas y principios de la OMC constituyen la base de las políticas y estrategias comerciales utilizadas por los Estados, sobre cuya base pueden reclamar ante el Órgano de Solución de Controversias de la organización en caso de que otros países las incumplan.

\section{Evolución de la participación de los países en desarrollo en el régimen internacional de comercio del GATT a la OMC}

La participación de los países en desarrollo en el régimen de comercio ha tenido altibajos. El primer fracaso de una activa participación de estos países se constató en los denominados "Acuerdos de Bretton Woods", los cuales, como ha sido mencionado, incluían la creación de una organización internacional para coordinar el comercio: la OIC.

Esta organización tendría como objetivos facilitar la liberalización del comercio y llamar la atención sobre la relación entre el comercio, el empleo y el desarrollo económico. En el marco de la OIC, el desarrollo económico sería una responsabilidad compartida entre los países industrializados y los países en desarrollo; asimismo, la OIC proveía a estos últimos la posibilidad de aplicar varias excepciones a las reglas de No Discriminación (Oatley, 2004). El Congreso estadounidense no aprobó la adhesión de su país a la OIC, lo que fue decisivo para su fracaso. En su lugar se estableció el GATT, que se convertiría en la base para la creación de la posterior OMC en 1995. 
Según Oatley (2004), el GATT integró los intereses de Estados Unidos y Europa Occidental, pero no consideró ni recogió las preocupaciones de los países en desarrollo; prueba de ello fue que la agricultura no fue incluida dentro del marco regulatorio del GATT, rubro en el que claramente tienen ventaja los países en desarrollo; lo mismo sucedió con los textiles (Acuerdo Multifribras). Según el mismo autor, el nuevo sistema se fundamentó en el principio de "igualdad" y no tomó en cuenta el desigual desarrollo económico entre países; sólo estableció unas pocas excepciones en el artículo XVIII.

De conformidad con dicho artículo, se permitía a los países en desarrollo apartarse de los compromisos arancelarios y utilizar ciertas ayudas del Estado con el fin de establecer una industria particular y/o para restringir las importaciones por problemas de balanza de pagos. Este artículo no incluía medidas para estimular el acceso a terceros mercados, particularmente de los países desarrollados, elemento de notable importancia para las economías de los países en desarrollo (Oatley, 2004).

Con el fracaso de la OIC, los países en desarrollo se convirtieron en críticos del GATT; creían que el sistema establecido (liberal y no discriminatorio) "limitaría su capacidad para desarrollar la industria manufacturera que ellos pensaban era central para el desarrollo económico" (Oatley, 2004, p. 29). Es así como iniciaron una batalla contra los países desarrollados con el fin de superar las limitadas excepciones a su favor estipuladas en el artículo XVIII (Oatley, 2004).

Según López-Jurado (2001), con el fin de la colonización tomó fuerza el debate sobre la relación entre comercio y desarrollo. Los países en desarrollo reclamaban un sistema más acorde a sus condiciones y manifestaban que "la desigualdad no se corrige mediante la aplicación de medidas idénticas para todos, sino mediante la adopción de un marco normativo que, favoreciendo a algunos países, pueda conducir a una cierta justicia distributiva” (López-Jurado, 2001, p. 4).
Desde la década de los 60’s, las demandas de los países en desarrollo empezaron a tomar fuerza. En 1961, en el marco del GATT, se adoptó la Declaración sobre la "Promoción del Comercio de los Países menos desarrollados”, que impulsó el acceso a los mercados para estos países. Asimismo, en la Reunión Ministerial del GATT de 1963 se estableció un Plan de Acción con medidas para favorecer el comercio de los países en desarrollo. Finalmente, en 1964, en Ginebra, tuvo lugar la Conferencia de Naciones Unidas para el Comercio y Desarrollo (UNCTAD, por sus siglas en inglés), la cual se convirtió en un órgano permanente.

La UNCTAD se encargó del mejoramiento del acceso a los mercados para los países en desarrollo. Para el efecto, esta organización logró incorporar al GATT la denominada Parte IV: "Comercio y Desarrollo", la cual instó a los países desarrollados a tomar acciones que reduzcan y eliminen las barreras a los productos de interés de los países en desarrollo.

La Parte IV codificó el concepto de "No Reciprocidad" en las negociaciones comerciales, propiciando el establecimiento en 1971 de los acuerdos comerciales entre países en desarrollo (OMC, 2009), así como del Sistema Generalizado de Preferencias (SGP), mediante el cual los países desarrollados otorgarían preferencias arancelarias unilaterales, temporales y sin exigencia de reciprocidad a los países en desarrollo (Di Giovan, 1992).

La denominada Parte IV incluyó las excepciones del artículo XVIII del GATT y, adicionalmente, permitió un acceso discriminatorio preferencial a terceros mercados para los países en desarrollo, lo cual constituía el fundamento del denominado Sistema SGP (López-Jurado, 2001). La instancia encargada de controlar la aplicación de la Parte IV sería el Comité de Comercio y Desarrollo. Por su parte, la decisión de permitir Acuerdos Comerciales entre países en desarrollo se convirtió en un fundamento jurídico en el GATT mediante el "Protocolo relativo a las negociaciones comerciales entre países en desarrollo", en el cual se afirmó 
que "la integración económica entre los países en desarrollo constituye un elemento importante en la estrategia internacional del desarrollo" (López-Jurado, 2001, p. 8).

En la Ronda Tokio (llevada a cabo entre 1973 y 1979) se emitió la Cláusula de Habilitación, la cual complementó al sistema SGP: "con la Cláusula de Habilitación se autorizó la concesión unilateral a los países en desarrollo de un trato aduanero preferencial, conforme con el Sistema Generalizado de Preferencias -SGP-” (Llorente, 2006, p. 273). Esta Cláusula fue instrumentada por medio de la decisión denominada "Trato diferenciado y más favorable, reciprocidad y mayor participación de los países en desarrollo".

El principal objetivo de esta Cláusula era aprobar una excepción al principio de Nación Más Favorecida (NMF), a fin de incrementar las oportunidades comerciales de los países en desarrollo (López-Jurado, 2001). La Cláusula de Habilitación consolidó el concepto de Trato Especial y Diferenciado (TED) y el principio de No Reciprocidad. Esta cláusula se constituyó en la base legal para el TED, el SGP y los acuerdos entre países en desarrollo (OMC, 2009).

A pesar de estos avances para los países en desarrollo, el GATT enfrentaba varios desafíos, limitaciones y problemas que minaban su credibilidad y efectividad. El autor Baracuhy (2013) señala que a pesar de los principios establecidos, el sistema de comercio se había convertido en una "alianza entre países desarrollados” (p. 344), pues no cubría los temas de interés de los países en desarrollo, tales como la agricultura y los textiles. Según el mismo autor, en general las rondas de negociaciones del GATT se habían concentrado en la reducción de aranceles, principalmente de productos industriales.

En este contexto, se consideró necesario iniciar un nuevo proceso de negociación, el cual duró varios años y concluyó con la creación de la OMC. Las negociaciones incluyeron nuevas temáticas, entre ellas la agricultura, ser- vicios, propiedad intelectual, entre otras. El propósito de la nueva organización era superar las limitaciones que se habían registrado en el GATT y crear un sistema de comercio más transparente y predecible.

A mediados de los 80, los gobiernos de varios países en desarrollo cambiaron sus estrategias de desarrollo y el comercio internacional pasó a formar parte de sus agendas (Oatley, 2004). Así, varios países se adhirieron al GATT y posteriormente a la OMC. En todo caso, el TED y el SGP, amparados por la Cláusula de Habilitación fueron, importantes conquistas alcanzadas por los países en desarrollo durante la existencia del GATT.

\section{Los países en desarrollo en la OMC: beneficios y limitaciones}

Como lo expresaran Keohane y Nye (1989), en gran medida la creación de instituciones como la OMC es un mecanismo efectivo para que países de menor tamaño, poder e influencia puedan lograr mayor participación y equidad, debido a que el poder de negociación se incrementa a nivel multilateral, ya sea por la formación de alianzas o por la presión conjunta sobre temas de interés común. En negociaciones bilaterales, los Estados más fuertes pueden más fácilmente imponer a los más pequeños condiciones y requerimientos que estos últimos se verán obligados a aceptar.

Sin embargo, también es cierto que determinados Estados (los de poder) pueden influir -y de hecho lo hacen- en las instituciones internacionales (Oatley, 2004). Toda organización internacional se crea sobre la base de intereses, al menos aparentemente comunes, por los cuales todos están motivados a cooperar. No obstante, las premisas realistas respecto a las manifestaciones de poder que inciden en los regímenes mantienen plena vigencia.

Esto ha sucedido en la OMC, pues se la ha criticado de no ser lo suficientemente justa y equilibrada y de responder a los intereses de los grandes países. Para Gilpin (2001), 
la OMC también es una creación estadounidense, ya que recogió los intereses de este país que se había convertido en proveedor de servicios y alta tecnología.

Como ha sido mencionado, la participación de los países en desarrollo en el sistema multilateral de comercio fue restringida durante la vigencia del GATT: "a lo largo del periodo de posguerra, los países industrialmente avanzados han dominado las negociaciones comerciales y como consecuencia han podido establecer agendas de negociación que reflejan sus intereses" (Oatley, 2004, p. 38). Así, las negociaciones comerciales se habían enfocado en la liberalización del comercio de bienes intensivos en capital y habían excluido el comercio en agricultura y bienes intensivos en mano de obra (Oatley, 2004).

No obstante, al menos teóricamente, con la creación de la OMC estos países han mejorado su posición y rol dentro del sistema. Es positivo el hecho de que se haya configurado una organización internacional con reglas, normas y principios establecidos (un régimen) que dirijan las relaciones entre sus miembros, tal como habían anhelado los países en desarrollo con la OIC.

Con la Ronda Uruguay, que tuvo lugar entre 1986 y 1994, y al final de la cual se creó la OMC, se afirmó el principio de No Reciprocidad, la Cláusula de Habilitación y el TED. La OMC amplió los objetivos del GATT, ya que integró la visión del desarrollo para asegurar la participación de los países en desarrollo en el comercio internacional; de esta manera, quedó reconocido el vínculo entre comercio y desarrollo (OMC, 2009).

Los países en desarrollo han ido participando de manera más activa en la OMC y constituyen la mayoría de los miembros. El ingreso a la OMC les ha concedido algunas ventajas. Según Oatley (2004), la mayor participación de estos países en el sistema multilateral de comercio se pudo evidenciar en la Conferencia Ministerial de Doha de noviembre de
2001, en la cual dichos países formaron coaliciones y diseñaron estrategias que les permitió incluso formar parte de la organización y dirección de la Conferencia.

Según el mismo autor, en dicha Conferencia los países en desarrollo también lograron resistir la presión europea para incluir negociaciones sobre reglas de inversión y política de competencia, insistiendo que antes de la incorporación de nuevos temas, los países avanzados debían liberalizar el comercio agrícola y de bienes manufacturados intensivos en mano de obra. En este sentido, el embajador de Brasil en Estados Unidos habría manifestado que "para que las negociaciones sean exitosas será necesario que los países industrialmente avanzados reconozcan la nueva composición de fuerzas en la OMC” (Oatley, 2004, p. 39).

El liderazgo de Brasil fue determinante en los años subsiguientes para cambiar la estructura de negociación de la Ronda de Doha; es así como en 2003 surgió formalmente el G20 como una nueva fuerza en las negociaciones, el cual se convertiría en uno de los bloques más significativos dentro de la OMC (Baracuhy, 2013).

Con el establecimiento del Programa de Doha para el Desarrollo, se incorporaron algunas disposiciones en favor de los países en desarrollo y de los países menos adelantados (PMA). Con respecto a los PMA ${ }^{1}$, en la Declaración de Doha se expresó el compromiso de mejorar el acceso a los mercados -libre de derechos y contingentes- para los productos originarios de dichos países. Asimismo, se acordó realizar un examen sobre la transferencia de tecnología para los países en desarrollo y se aprobó una decisión relativa a las dificultades que enfrentan estos países para la implementación de los Acuerdos de la OMC (OMC, 2009).

Otros temas de discusión que se han incorporado a los debates en favor de los países en desarrollo han sido la situación de las peque-

1 Párrafo 42 y 43 de la Declaración de Doha para el Desarrollo. 
ñas economías, que enfrentan problemas específicos para su participación en el comercio mundial, e iniciativas como la de "Ayuda para el Comercio", programa lanzado en 2005 para ayudar a los países en desarrollo a desarrollar los conocimientos y la infraestructura necesarios para el comercio (OMC, 2009).

La OMC es el foro de negociación en el cual los países en desarrollo han podido exponer sus intereses y preocupaciones y presentar reclamos por eventuales violaciones a la normativa OMC a través del Mecanismo de Solución de Disputas. La OMC ha permitido la formación de coaliciones y estrategias de negociación entre los países más débiles, a fin de defender sus intereses comunes e incrementar su poder de negociación.

Los países en desarrollo reciben en el marco de la OMC un TED, según el cual los países desarrollados tienen la posibilidad de otorgar a los países en desarrollo un tratamiento más favorable que a otros miembros de la OMC. Algunas de dichas disposiciones son: plazos más largos para el cumplimiento de los compromisos, ciertas excepciones a las reglas, disposiciones para aumentar el acceso a los mercados, consideración especial de su condición en la aplicación de medidas antidumping, salvaguardias y obstáculos técnicos al comercio, asistencia y cooperación técnica, entre otros (OMC, 2009). La aplicación del TED, así como de todos los asuntos relacionados con el desarrollo, está a cargo del Comité de Comercio y Desarrollo.

El TED es un principio que forma parte integral de los Acuerdos de la OMC. Todas las negociaciones de la Ronda de Doha deben incorporar este principio. El TED, que no existió en los primeros años del GATT, empezó como un reconocimiento de la necesidad de proveer flexibilidades a los países con bajos estándares de vida y en etapas tempranas de desarrollo. Este reconocimiento, registrado en el artículo XVIII del GATT, se extrapoló a los Acuerdos de la OMC (OMC, 2009). El TED es un instrumento útil que reconoce las asimetrías económicas y de desarrollo entre países.

En la Declaración de Doha, los países miembros convinieron en examinar todas las disposiciones sobre TED para fortalecerlas y "hacerlas más precisas"2 y se encargó al Comité de Comercio y Desarrollo que identificara las disposiciones sobre TED de carácter obligatorio y considerara las consecuencias de convertir en obligatorias las que aún no lo eran (OMC, 2009).

A pesar de todas las ventajas y preferencias señaladas, también existen limitaciones que los países en desarollo enfrentan en el marco de la OMC. Según George (2002), algunas críticas a la OMC son: el déficit democrático o la falta de información a la sociedad que habría motivado las protestas de ONGs durante la Conferencia Ministerial en Seattle en 1999, la aplicación de los principios de Nación más Favorecida (NMF) y Trato Nacional (TN) sin considerar las condiciones de producción (explotación laboral y degradación del ambiente), el excesivo poder del Órgano de Solución de Diferencias (OSD) que tendría excesiva discrecionalidad en la interpretación de los acuerdos, los principios y normas difusos y ambiguos y muchas veces meramente retóricos (por ejemplo, los principios sobre desarrollo sostenible están sólo en el preámbulo), y la excesiva cesión de la soberanía estatal.

Por otra parte, la misma autora manifiesta que muchas disposiciones en materia de TED no constituyen una obligación, pues se aplican "en la medida de lo posible", son temporales $\mathrm{y}$ en muchas ocasiones se aplican marginalmente, por lo que se ha cuestionado su efectividad. Adicionalmente, aún existen subsidios, medidas arancelarias y cada vez crecen más las medidas no arancelarias que restringen las posibilidades de comercio de los países en desarrollo.

George (2002) hace notar que a pesar de que

2 Párrafo 44 de la Declaración de Doha para el Desarrollo. 
el artículo XVI del Acuerdo de Marrakech se acoge a las disposiciones del GATT en relación al tratamiento de los países en desarrollo, también se establece que esto se hará "salvo disposición en contrario de los Acuerdos de la OMC”, los mismos que urgen a los países en desarrollo a incorporarse a las normas generales de la OMC, lo que en sí constituye una limitación ya que los países en desarrollo presentan mayores dificultades para la aplicación de las obligaciones que estipula la OMC debido a su reducido desarrollo.

El debate actual giraría en torno a si es que la OMC es accesible para los países en desarrollo en la misma medida que para los países grandes, pues los países con limitados recursos económicos y de capacidad enfrentarían dificultades para manejar negociaciones complejas o beneficiarse del sistema de solución de controversias (Bayne, 2013).

Los marcados intereses y desacuerdos entre los países no han permitido avanzar en temas fundamentales en el marco de la Ronda de Doha. Muchos consideran que el estancamiento de dicha ronda ha mermado la efectividad y profundizado la desconfianza en la OMC. En la Conferencia Ministerial, llevada a cabo en Nairobi en el año 2015 a pesar de haberse alcanzado algunos acuerdos sobre la reducción de subsidios a la exportación de productos agrícolas y algunas disposiciones positivas para los PMA, se habría evidenciado la imposibilidad de lograr un consenso en torno a la Agenda de Doha.

Así lo habría expresado el embajador de los Estados Unidos ante la OMC, Michael Punke, durante el Examen de las Políticas Comerciales de ese país en el marco de dicha organización, llevado a cabo en diciembre de 2016, durante el cual habría hecho un llamado a girar la página de Doha y a concentrarse en "cuestiones comerciales modernas" que verdaderamente se ajusten a la realidad del mundo actual, pues, a su criterio, esa sería la manera de fortalecer el sistema multilateral de comercio (Caporal, 2016).
La política comercial que adopte la nueva administración estadounidense podría profundizar los desacuerdos en la OMC. Durante la campaña electoral, el entonces candidato Donald Trump emitió polémicos pronunciamientos en torno a la política comercial que implementaría durante su Gobierno y, entre otras cosas, llegó a amenazar con una eventual salida de los Estados Unidos de la OMC. Las designaciones de autoridades en materia comercial realizadas hasta el momento dan cuenta de la postura que adoptará el Gobierno de Donald Trump en este ámbito.

La "potencial” muerte de la Ronda de Doha constituirá un fracaso para los países en desarrollo, pues significará la eliminación en la Agenda multilateral de comercio de significativos temas relacionados al desarrollo, por los cuales estos países trabajaron por años con significativos resultados. Parecería que los países desarrollados estarían buscando cambiar la tónica de la agenda multilateral e incorporar temas que aparentemente "respondan a las nuevas necesidades o realidad”, sin tomar en cuenta -nuevamente- que las necesidades y realidades son distintas entre países desarrollados y en desarrollo.

Expertos consideran que el fracaso de la Ronda de Doha habría contribuido a la proliferación de acuerdos comerciales bilaterales, regionales y hasta interregionales. Para algunos, dichos acuerdos serían herramientas para continuar liberalizando el comercio y alcanzar acuerdos en determinadas temáticas que habrían encontrado obstáculos a nivel multilateral; para otros, por el contrario, la proliferación de dichos acuerdos constituirían un peligro para el sistema multilateral ${ }^{3}$.

\footnotetext{
3 Existen básicamente dos líneas de pensamiento en torno a este debate: aquellos que consideran que el regionalismo contribuye al multilateralismo ("building block"), cuyo principal representante es Hufbauer, y aquellos que, por el contrario, creen que el regionalismo obstruye o mina al multilateralismo ("Stumbling block"), con Bhagwati a la cabeza. Para los primeros, los acuerdos comerciales preferenciales son una alternativa inmediata a los acuerdos multilaterales para el libre comercio; por su parte, para Bhagwati, los acuerdos comerciales crean áreas de exclusión con
} 
Se ha reconocido la importancia de muchos de dichos acuerdos por el posible impacto en el comercio internacional (y en el sistema multilateral de comercio) dado el número de países que participan y el tamaño de sus mercados. El Acuerdo de Asociación Transpacífico (TPP, por sus siglas en inglés) ha sido precisamente uno de los acuerdos considerados de mayor envergadura, el cual, sin embargo, parecería empezar a diluirse ante la incierta posición que finalmente adopte la nueva administración estadounidense en torno a este tema.

\section{CONCLUSIONES}

El liderazgo, la cooperación y el consenso ideológico son elementos fundamentales para que un régimen -como el de comercio- y una organización -como la OMC- funcionen. El régimen internacional de comercio no ha funcionado solamente por el liderazgo de ciertas potencias; sin duda el rol de las grandes hegemonías ha sido determinante en la conducción de la OMC; no obstante, sin cooperación dicho liderazgo no habría sido sostenible. Asimismo, el consenso ideológico es fundamental para sostener un régimen, pues si solamente pocos imponen reglas los demás no tendrán motivación para permanecer en él. Por el contrario, si están convencidos de sus ventajas, contribuirán a mantenerlo.

La OMC ha proporcionado beneficios al sistema multilateral de comercio. La creación de reglas ha generado confianza entre los países y, por ende, estabilidad, transparencia y mayor previsibilidad. El hecho de contar con un foro para negociaciones y para la resolución de disputas es importante. Sin embargo, la OMC enfrenta aún varias limitaciones y desafíos.

El sistema multilateral de comercio ha otorgado múltiples beneficios y derechos a los países

sistemas preferenciales complicados, discriminatorios, proteccionistas y a veces hasta contradictorios (un "Spaghetti bowl") que obstaculizan el libre comercio y minan el sistema multilateral. Ver más sobre este debate en: De Lima-Campos, A. (2009), p. 102-103. en desarrollo. Un sistema de reglas establecidas es positivo para que estos países mejoren su poder de negociación, comparado con los mecanismos disponibles a nivel bilateral. Los países en desarrollo han trabajado con el fin de alcanzar un trato especial y diferenciado en el marco de la OMC y han conseguido poner sobre la mesa de discusión muchos temas de su interés.

La Ronda de Doha incorporó positivas temáticas para estos países, sin embargo no ha sido posible alcanzar un consenso sobre ellos en ya casi dieciséis años. El estancamiento en las negociaciones de dicha Ronda y su potencial finalización sin acuerdos podría significar un considerable fracaso para los países en desarrollo. Parecería ser que se estaría buscando reemplazar la agenda de Doha por una agenda con "modernos" temas que, sin embargo, podrían no necesariamente responder a la realidad de la mayoría de los países en desarrollo.

Si bien es cierto que la OMC no ha sido todo lo equilibrada y justa que debería ser, es mejor a la unilateralidad para los intereses de países pequeños. A pesar de las limitaciones que pueden presentar las organizaciones internacionales, éstas constituyen foros de participación para países débiles. El contar con un marco normativo establecido es una ventaja para estos países, pues las decisiones se toman con arreglo a dichas normas y no conforme a las decisiones "azarosas" de ciertos países. No obstante, los intereses de los grandes países siempre priman y, en última instancia, configuran las normas establecidas en las organizaciones.

Para los países en desarrollo constituye un desafío convertir a las organizaciones, como la OMC, en el instrumento o mecanismo más idóneo para la defensa de sus intereses, para lo cual es necesario conocer el funcionamiento de las organizaciones, su estructura y mecanismos disponibles a fin de, sobre la base de ello, definir las mejores estrategias para su participación en las mismas. 


\section{REFERENCIAS BIBLIOGRÁFICAS}

Attiná, F. (2001). El Sistema Político Global, Barcelona, España: PAIDOS.

Baylis, J., Smith, S., y Owens, P. (2008). The Globalization of World Politics. Nueva York, Estados Unidos: Oxford University.

Bayne, N., y Woolcock, S. (Ed). (2013). The New Economic Diplomacy: Decision-Making Negotiation in International Economic Relations. Londres, Gran Bretaña: The London School of Economics and Political Science ASHGATE

Bayne, N. (2013). Plurilateralism and Multilateralism: Comparing Institutions. En Bayne, N., y Woolcock, S. (Ed). The New Economic Diplomacy: Decision-Making Negotiation in International Economic Relations. Londres, Gran Bretaña: The London School of Economics and Political Science ASHGATE.

Baracuhy, B. (2013). Brazilian Economic Diplomacy: Agriculture and the WTO. En Bayne, N., y Woolcock, S. (Ed). The New Economic Diplomacy: Decision-Making Negotiation in International Economic Relations. Londres, Gran Bretaña: The London School of Economics and Political Science ASHGATE

Caporal, J. (2016, 19 de diciembre). Punke calls for moving past Doha, warns of over-reaching Appellate Body. Inside US Trade. Recuperado de https:// insidetrade.com/daily-news/punke-calls-moving-past-doha-warns-over-reaching-appellatebody?utm_source $=$ dlvr.it\&utm_medium $=$ twitter

De Lima-Campos, A. (2009). Preferential Trade Agreements. Trade and International Negotiations for Journalists. Río de Janeiro, Brasil: CEBRI. Recuperado de http://rss.cebri.org/jornalistas_cebri\%5B1\%5D.pdf

Di Giovan, I. (1992). Derecho Internacional Económico y relaciones económicas internacionales. Buenos Aires, Argentina: Abeledo Perrot.

Dunne, T., Kurki, M., y Smith, S., (2007). International Relations Theories. NueTTva York, Estados Unidos: Oxford University.

Espino, M. (1998). La Organización Mundial del Comercio y sus instrumentos normativos. Bogotá, Colombia: Ediciones Jurídicas Gustavo Ibáñez Ltda.

Fernández, J. C. (2001). Sistema del Comercio Internacional. España: Civitas.

George, S. (2002). Pongamos a la OMC en su sitio. Barcelona, España: Editorial Icaria, 1º Edición.

Gilpin, R. (2001). Global Political Economy. Estados Unidos: Princeton University Press.
Herdegen, M. (1998). Derecho Económico Internacional. Bogotá, Colombia: Konrad Adenauer, $2^{\circ}$ Edición.

Hasenclever, A., Mayer, P., y Rittberger, V. (1999). Las teorías de los Regímenes Internacionales: Situación actual y propuestas para una síntesis. Revista FORO Internacional. México DF, México. Volumen 39, Número 4.

Keohane, R. (1993). Instituciones Internacionales y Poder Estatal. Buenos Aires, Argentina: Grupo Editor Latinoamericano.

Keohane, R., y Nye, J. (1989). Power and Interdependence: World Politics in Transition. Boston, Estados Unidos: Harvard: Harper Collins Publishers.

López-Jurado Romero De La Cruz, C. (2001). El tratamiento de los países en vías de desarrollo en la Organización Mundial del Comercio y las iniciativas unilaterales de la Comunidad Europea. Revista Electrónica de Estudios Internacionales. Recuperado de www.reei.org

Llorente, G. (2006). Conflicto entre las normas que regulan la Organización Mundial de Comercio y las que regulan la Integración, ¿cuáles prevalecen?. Revista de Derecho FORO. Quito, Ecuador: Corporación Editora Nacional.

Oatley, T. (2004). International Political Economy. Estados Unidos: Pearson Education.

Organización Mundial de Comercio (OMC). (2001). Declaración de Doha para el Desarrollo. Recuperada de: https://www.wto.org/spanish/thewto_s/ minist_s/min01_s/mindecl_s.htm

Organización Mundial de Comercio (OMC). (2003). Los Textos Jurídicos, Los Resultados de la Ronda Uruguay de Negociaciones Comerciales Multilaterales. Ginebra, Suiza: OMC.

Organización Mundial de Comercio (OMC). (2004). El futuro de la OMC: una respuesta a los desafíos institucionales del nuevo milenio, Ginebra, Suiza: OMC.

Organización Mundial de Comercio (OMC). (2005). Entender la OMC. Ginebra, Suiza: OMC, $3^{\circ}$ Edición. Recuperado de: https://www.wto.org/spanish/thewto_s/whatis_s/tif_s/dev1_s.htm

Organización Mundial de Comercio (OMC). (2006). Documento de trabajo: Aspectos de las negociaciones de la Ronda de Doha relativos al desarrollo. Ginebra, Suiza. OMC - Comité de Comercio y Desarrollo. Recuperado de WT/COMTD/W/143/Rev.2

Organización Mundial de Comercio (OMC). (2009). Multilateral Trade Agreements. Ginebra, Suiza: Cursos de formación OMC. Recuperado de https://ecampus. 
wto.org/search.asp?lang=En

Organización Mundial de Comercio (OMC). (2015, 19 de diciembre). Los miembros de la OMC logran un Paquete de Nairobi "histórico" para África y para el mundo entero. Recuperado de https://www.wto. org/spanish/news_s/news15_s/mc10_19dec15_s. htm

Rojas, F. (2000). Multilateralismo, perspectivas latinoamericanas. Santiago, Chile: FLACSO Nueva Sociedad.
Salgado, W. (1996). El Sistema Mundial de Comercio, el Ecuador frente al GATT y OMC. Quito, Ecuador: Corporación Editora Nacional.

Smith, S., Hadfield A., y Dunne, T. (2008). Foreign Policy Theories. Actors. Cases. Estados Unidos: Oxford University Press.

Tamames, R. (1999). Estructura económica internacional. Madrid, España: Alianza Editorial. 19 Edición. 